\title{
Radiology and primary care in Europe
}

\author{
The Executive Council of the European Society of Radiology \\ (ESR) and the Council of the World Organization of National \\ Colleges, Academies and Academic Associations of General \\ Practitioners/Family Physicians/ European regional branch \\ (WONCA Europe)
}

Received: 10 May 2010 /Accepted: 11 May 2010 /Published online: 11 June 2010

(C) European Society of Radiology 2010

\begin{abstract}
This joint paper outlines the current status and future outlook of the cooperation between radiology specialists and general practitioners. It discusses issues of referrals in primary care, imaging requirements, quality of care, types of radiology services and finances, all seen by both sides - radiologists and primary care physicians.
\end{abstract}

Keywords Radiology Primary care $\cdot$ Family care . Guidelines · Access · Quality

\section{Introduction-the role of primary care and general practice in health care delivery}

Primary care may be considered the point of first contact for patients with a doctor and the type of clinician concerned is dependent on the definition used for primary care. According to the European definition General Practice/ Family Medicine (GP/FM) is "the first medical contact within the health care system, providing open and unlimited access, dealing with all health problems regardless of the age, sex, or any other characteristic of the person concerned" (WONCA Europe, http://www. woncaeurope.org).

Although this central role of general practice in health care is generally accepted in some healthcare systems, the initial contact of a patient may also be a self referral to a specialist that the patient believes is appropriate because of a special symptom or status such as pregnancy. The initial

European Society of Radiology $(\bowtie)$

Neutorgasse 9/2,

AT-1010 Vienna, Austria

e-mail: communications@myESR.org care of this patient is then undertaken by the specialist who may refer the patient on to another specialist if the condition is not one that is in his/her field. Patients may also present initially at an emergency department with ailments that do not require immediate admission to the hospital, because they do not have access to any other primary care service, and are therefore treated over a short term by those departments. On the other hand patients may sign up with a single or with a group of primary care physicians, a term which is used in this document identically with the term General Practitioners (GPs), who provide a continuous, comprehensive and coordinated care and are responsible for diagnosis and treatment of common ailments and for referral to secondary care when necessary, as clearly indicated by the European definition [1].

This paper is primarily devoted to this third scenario although allowances must be made for the considerable variation in the concept and structure of primary care services for patients across Europe. In some countries primary care services delivered by GPs is as yet less developed and only deal with cases of minimal every day disorders while anything that is more complex is dealt with by a specialist working in outpatient clinics or hospital environments to whom either the GP or the patient self refers. It is not the first time that GPs and Radiologists have produced a joint statement: the Royal College of GPs and the Royal College of Radiologists emphasised in 1993 and re-emphasised in 2004 the importance of imaging for the management of the patient in the primary care setting [2]. Now, due an initiative undertaken by the European Society of Radiology, this joint paper with the WONCA Europe underlines the need to see clinical radiology and primary care physicians to work together with the main aim to improve the care of patients in Europe. 


\section{Primary care in Europe}

The model of a comprehensive structure of doctors who are responsible for the health care of groups of people over a long term with a detailed knowledge of their patient's health records is well established in many European countries. These primary care physicians have to make the initial evaluation of the patient and instigate appropriate treatment. In doing so, GPs have a specific decision process determined by the prevalence and incidence of illness in the community and by the pre-and post-test probabilities using simple and inexpensive diagnostic tools. When there is uncertainty over the diagnosis or where the treatment required is more complex they are responsible for referring patients to specialists and for providing follow-up care after discharge.

In some countries this model is managed and funded directly by the state (for example in the UK) whereas in other countries it is independent of state management and funded either privately or through a healthcare insurance scheme, which may be state or independently resourced (for example in Austria). A recent development in some countries, especially in large cities with a mobile population, is a primary care service being delivered by walk-in centres for immediate care for common ailments ; however, these may lack the knowledge of the patient and the long-term commitment to continuous, comprehensive and coordinated care that is considered the hallmark of many primary care services. In one country in Europe health insurance organisations are allowed to offer contracts at reduced costs if patients agree to first contact primary care physicians in order to reduce specialist visits and the associated costs (it is true for Germany, however it has just began to be implemented). However, even in health care systems that provide open access to specialists, the first contact for patients is most often a GP.

\section{Referral from primary care to secondary care}

Primary care physicians require a broad knowledge of medicine: they are faced with illness which presents in an undifferentiated and unspecific way or at an early stage in its development some of which require urgent intervention [1]; they manage long-term problems and chronic care of patients such as diabetes and heart disease and direct the patient to the appropriate specialty service as need arises. This enables the primary care physician to diagnose and treat most common ailments without recourse to expensive secondary and tertiary hospital based services. The demographic changes with an increasing proportion of old patients with chronic and multiple diseases and who wish to be treated in their own environment also increase the need for enhanced primary care services.

It also empowers them to investigate a patient more fully in conjunction with the clinical radiologist prior to referring to another specialist, which may either avoid a referral or may result in a specific referral to the appropriate specialist thereby keeping delays to a minimum prior to treatment. This also increases the efficiency of hospital outpatient facilities and safes time and cost to the patient. It is also in agreement with the second core characteristic of the WONCA Europe definition that states that "GP/FM makes efficient use of health care resources" $[1,3] \ldots$ For such a means of referral to be effective the imaging would need to be organised by the clinical radiologist to ensure that the appropriate investigations are performed and to avoid repetition of investigations. In order to provide comprehensive care to take place in the primary care setting, access to a wide range of services is necessary. These include pathology, biology, imaging and also practice and community nursing services.

There is also potential for primary care physicians and GPs to refer patients directly to imaging departments for imageguided procedures - both diagnostic and therapeutic - on an out-patient, day case or in-patient basis. Thus, good collaboration between them and clinical radiologists is a need and a challenge at the same time. It serves both clinical disciplines and their patients: GP/FM that currently aims to refine its targets and strategy [4] and Radiology where a multispecialty collaboration has been seen as key to the future of the discipline [4].

\section{Imaging requirements of primary care}

In order to fulfil the above objectives it is necessary to address the requirements of patients and physicians in the primary care setting. Some clinical presentations in primary care are common and appear to be easily diagnosed clinically requiring no more therapy than reassurance and proprietary medicines available in all pharmacies by direct purchase. However, some of these ailments may present unusual or worrying features or are out of character for the individual patient all of which require further investigation.

Some conditions may be diagnosed or strongly suggested clinically but require imaging to confirm the diagnosis and to assess the extent of the changes. These are then treated appropriately and follow up examinations may be required to confirm or assess the degree of resolution. Sometimes the primary care clinician may be treating a patient with a confirmed diagnosis but resolution is slow or has failed and imaging is required to exclude or define complications. Finally, imaging may be undertaken for more complex cases that require specialist referral but prior investigations in conjunction with the clinical radiologist and the relevant specialist is undertaken to ensure that the referral is to the correct specialist and that the consultation is productive.

The types of investigation available to primary care clinicians should be based on relevance to the clinical 
problem and patient management and not only on the cost or complexity of the imaging procedure. This is also reflected in the European definition that mentions that GP/ FM "has a specific decision-making process determined by the prevalence and incidence of illness in the community" However, in some countries the access to diagnostic imaging services is limited to basic conventional examinations. Plain radiography should be provided and chest, bone and joint imaging is widely utilised by primary care services. Chest radiology is one of the most widely used diagnostic imaging techniques and it has been reported to change patient management by the GP in $60 \%$ of patients, to substantially reduce the number of referrals and initiated or changed therapy. It has also been shown to be cost effective [5]. Joint disease is also an important reason for consultation in general practice and although clinical history and examination can assist in the diagnosis of osteoarthritis radiographic demonstration may be required and the x-ray features have been shown to affect management regardless of the clinical picture $[6,7]$.

Developments in Computed Tomography (CT), Magnetic Resonance Imaging (MRI) and Ultrasound (US) have resulted in these modalities also being used extensively and should be essential services to be provided in Primary Care. Unfortunately, access may be limited by the number of more complex systems in some countries and GPs may not be allowed to refer patients directly. However, Magnetic Resonance is now the investigation of choice in many musculoskeletal, spinal and neurological complaints. The use of these modalities in primary care has not been studied scientifically in any detail and there remains a level of debate. A recent study on open GP referral for neuroimaging has shown that overall $48 \%$ of examinations demonstrated a significant abnormality and was considered by the GPs to have influenced management in $90 \%$ of patients [8]. The value of lumbar spine MR in back pain is still subject to debate but lumbar radiography or MR is reported to increase patient reassurance and satisfaction [9, 10]. Patients with knee pain are commonly seen by GPs and access to MRI for patients presenting to GPs with continuing knee pain has been shown to represent a cost effective use of health service resources [11]. Despite the absence of detailed evaluation it is clear that these investigations should be accessible to primary care, using the same referral guidelines as secondary care, although in the short term this may be dependent on availability and the allocation of resources. There is some evidence that the introduction of Radiologists' guidelines on referral has reduced the rate of referrals [12].

In some situations a single imaging procedure will avoid the need for further investigation and referral to a specialist or hospital, which would cost considerably more and may still involve the cost of the imaging examination. Increas- ingly complex studies including some MR and nuclear medicine examinations are more likely to be relevant to the specialist and recognition of availability of some studies may require limitation to specific users.

In order for these imaging examinations to be used effectively the primary care physician should communicate formally with the clinical radiologist outlining the clinical history and potential diagnosis in order for the radiologist to justify the investigation of choice in line with EU 97/43 Euratom Directive [13]. Where the investigation requested is considered inappropriate or where a more sophisticated but costly examination is preferred a direct discussion between the primary care physician and the clinical radiologist is of great importance. This, however, is unlikely to be possible on a regular basis due to time constraints of both parties and, therefore, referral guidelines which may be European or national should be utilised by the primary care physician to minimise inappropriate requests. The radiological report should not only describe the findings and potential diagnoses but should recommend further investigations, including the rationale for the recommendations, and whether more complex and expensive investigations should be undertaken. In some cases this may be followed by a verbal discussion. It is important for the radiologist to identify in the report any positive results that would appear to be irrelevant to the main diagnosis or treatment. It must be recognised that in some cases where a positive investigation result seems unlikely to the radiologist, a negative result is of great value to the primary care physician in reassuring the patient.

Some interventional procedures may also be undertaken on the basis of a direct referral to the interventional radiologist from primary care physicians. These may be performed on an outpatient or day-case basis with the post procedural care being shared by the radiologist and the primary care physician. In these circumstances the patient will be under the care of the clinical radiologist who will deal with most of the clinical workup and immediate follow-up. For that the clinical radiologist will have clear protocols in place to deal with complications that may occasionally arise. However, in a number of countries radiologists do not have the facilities to take care of their patients after the intervention and a change in this situation should be encouraged.

\section{Quality of care, issues for primary care physicians}

\section{Timing of service}

Some conditions managed by primary care physicians are acute and require rapid investigation. The imaging service must be in a position to respond by providing an urgent appointment with an immediate report transmitted to the 
primary care physician verbally, electronically or by fax, dependent on the systems available. Imaging of the chest in suspected pneumonia will be of little use if the patient has to wait a week for the chest radiograph and /or its result.

Many conditions, however, are chronic and the timing and speed of the investigation is not critical. These investigations can be performed more easily to suit the patient, particularly if they are working although the result should be efficiently transmitted to the primary care physician in order to allay any anxiety that the patient may have regarding the outcome. It is important also that unexpected findings, which may require more rapid clinical intervention should be transmitted directly to the primary care physician preferably by direct communication.

\section{Comprehensiveness of service}

It is important that the radiology report is accurate and comprehensive not only identifying the lesions but also providing advice on the significance of the findings and providing guidance for further investigation or referral. Many findings on imaging are the result of the normal aging process. Other findings may be anomalies of a benign nature which have no relevance clinically and some abnormalities seen may not be relevant to the clinical problem being investigated but are important to note.

\section{Clarity of reports}

The primary care physician may not be familiar with some of the radiological terminology and a clear decisive report is important. The reporting style may need to be modified accordingly. In some countries the patient has direct access to the report transmitted to the primary care physician which means that technical and clinical explanations must be included in the report as well as clear conclusions and advice for strategy. In certain systems the primary care physician has limited access to the images and, therefore, relies on the report to a greater extent than specialist colleagues. This may, however, change with internet access to digital images.

\section{Professional communication}

It is important that personal contact between the primary care physician and the clinical radiologist is encouraged so that advice can be given about the value of the initial investigation, the need for further appropriate investigations and the clinical implications of the radiological findings. A fair partnership in this communication should overcome traditional references of specialists when they invite to collaborate with GPs [14]. In some circumstances where an acute problem is identified direct referral to another specialist by the clinical radiologist may be required. This should be done in consultation with the primary care physician

\section{Types of radiology services for primary care}

\section{Outreach clinical radiology services}

In rural areas, comprehensive radiology departments may not be available within easy access but local community hospitals or primary care centres (for example in Greece) will have some imaging facilities used by primary care physicians. It is important that these facilities should be directly related to, or under the organisational control of a clinical radiologist. This is important to ensure that the requirements of the EURATOM directive on Ionising Radiation are implemented and that there is proper quality control and trained staff. The primary care physician should be aware of their role as 'referrer' and be prepared to answer queries from the 'operator' (usually a radiographer) and the 'practitioner' (usually a radiologist). Where possible there should be direct picture archiving and communications system (PACS) linking to a central department where the images can be reported efficiently and effectively. In these circumstances it is important for the reporting radiologists to have a dialogue with the rural primary care physicians and organised visits by the clinical radiologists and rotation of staff in and out of the local community hospital departments or rural primary care centres would also enhance clinical governance and training.

In some countries clinical radiologists operate their own small units providing basic or even advanced imaging services to both primary care physicians and specialists working nearby at the community level (for example in Austria). Although such a system can be efficient there will inevitably be some limitation on the range of examinations available in small peripheral units; further, the necessary support should be available if intravenous injections of contrast medium, which are associated with potentially lethal complications, are undertaken. There will be clinical and cost effective barriers to the provision of complex computerised imaging examinations; a close working relationship between the peripheral units and the main Radiology Department will help to manage further investigation efficiently for the patient and enhance the quality of care with good communications between the clinical radiologist and the patient's primary care physician. 
Imaging services provided in primary care facilities

Imaging services for primary care should usually be provided in Radiology Departments where there is a range of equipment and fully trained expertise. However, in some countries (for example in Greece) imaging services such as basic radiography and ultrasound have been established in some large primary care centres in rural areas. This may be driven by a desire for patients to receive more routine imaging close to home, which may be valuable for rural communities. There may also be a financial advantage to the practice in some countries with personal insurance-based systems. Although there is some evidence that Australian doctors serving rural and remote areas have undertaken professional development and quality assurance activities in radiology after education [15] it is unlikely, however, that most centres will have the necessary expertise to deliver these services unless this is provided as an out reach service by a Radiology Department. It is particularly important that all staff members involved in undertaking and interpreting the examinations are properly trained to a similar standard as those in the Radiology Departments that would otherwise be providing the service. They must be suitably qualified, competent and experienced to perform each separate procedure which they are undertaking. They should be performing a sufficient number of examinations to maintain their skills and they should also maintain their continuing medical education in this field. It is also important that the equipment and facilities used are fit for purpose. If radiographic services are provided in the primary care setting they must comply with all national and European legislation.

It must be recognised that self referral (from clinicians to themselves as imagers) has been shown to be more expensive in health care provision; thus, all referrals by primary care physicians should be in line with the referral guidelines published by the EU or by national radiological organisations [16, 17]. The provision of these services within primary care setting should represent value for money and should be subject to external audit and monitoring for quality, clinical effectiveness and cost which means that if expensive equipment is installed in primary care facilities it must be fully and efficiently utilised. Ultimately, it is the interpretation of the examination that is key to patient care and the patient has a right to expect a high standard of reporting skill commensurate with the investigation being undertaken.

\section{Teleradiology services}

The increased development of national and international teleradiology services and the ability of complex equipment to be transported to the patient is a major development for primary care imaging provision. Mobile CT, MRI, US and Positron Emission Tomographic (PET) equipment is now available throughout Europe. These can be sited on a regular or intermittent basis in easy reach of primary care facilities enabling patients to be scanned closer to home in areas with population densities that are insufficient to support a fixed site scanner. The images are transmitted to reporting centres and the reports transmitted back to the primary care physicians. These services are invariably provided by private or public companies and the reporting undertaken either in centrally located reporting centres in Europe or, even outside Europe with the consent of the patient, or by individual radiologists as private contractors to the mobile imaging company. It is also possible that large hospitals may provide similar outreach mobile services which are serviced from the main Radiology Department of the hospital. Finally, national health services may purchase such services directly from companies to provide the required direct imaging access to primary care.

While this has advantages of convenience for the patient it may result in a reduced quality of service to the primary care physician unless there is close liaison beween the Radiologists providing the report and the referrer. This has already been demonstrated in some government led projects.

It is of particular importance for primary care physicians that opportunities are available to discuss the appropriate investigation for their patient, the implications and relevance of the imaging findings, any further investigations that may be required and the impact of the imaging on the future management of the case. Although this is difficult for an international teleradiology service to provide especially if there are language differences between the reporting centre and the primary care facility, there is some evidence that telecardiology improves the decision making of general practitioners, reducing cost and time $[18,19]$

Financial issues for the provision of radiology services to primary care

There are differing methods of funding health care provision throughout Europe. This will impact on the varying ability to provide radiology services to primary care physicians. In some EU countries where individual's health care is paid through an insurance system direct recompense can be made to the Department of Radiology by the patient's insurance without impact on the primary care physician (Austria). Systems may exist that provide the financial resources directly to primary care physicians to purchase the required imaging for their patients (UK). In 
these circumstances the amount of resource provided per patient is crucial as insufficient funding will remove the incentive for primary care physicians to order the imaging themselves resulting in greater referral to hospitals or specialists in order to defer the cost. Other countries may have central purchasing agencies that agree contracts with Radiology Departments on behalf of primary care physicians which may be on a block contract or payment by case (USA). Finally, Radiology Departments may be funded directly through a budget provided by the hospital who receive their income either as a block allocation or on a case by case basis from both primary and secondary care as part of a direct DRG payment.

There may be a number of variations on these models. In order to stimulate direct referral from primary care it is important that a financial system is in place that does not penalise the primary care physician and which recognises that primary care radiology is both cost effective and efficient for the patient: it reduces unnecessary referral to expensive hospital outpatient depart- ments and will result in a more efficient service as the patient will arrive at the outpatient departments more effectively evaluated.

\section{Conclusion}

It is obvious that, as technology in Radiology advances, standards of care improve and health care systems develop, the imaging needs of patients and their primary care physicians change. Furthermore the provision of imaging services throughoutEurope varies considerably. As a consequence, the professional collaboration between primary care physicians (especially GPs) and radiologists/radiology services should be newly defined and harmonized where possible. This paper provides some background information and key messages for consideration (box).

\section{Highlights of this paper}

1. The provision of a timely and comprehensive imaging service for primary care enables patients to be treated in the community where appropriate or to be efficaciously referred to secondary care. Providing services to emergency primary care and accident and emergency units is not covered by this paper as it necessitates a different type and level of service.

2. It is essential that GPs and primary care physicians to make efficient use of the available health care resources and provide coordinated and integrated health care in the primary care setting considering referral to radiologists when evidence-based appropriateness criteria are satisfied. These criteria should be part of protocols endorsed by both involved groups. GPs and primary care physicians should provide a clinical informative report when they refer their patients to radiologists.

3. It is essential that clinical radiologists and Departments of Radiology provide a high quality, efficient and cost effective service which satisfies the requirements of the patients and the primary care physician who is responsible for the continuity of care. It is important for individual Departments of Radiology to define a strategy for the type and level of service and its implementation with their referring primary care physicians.

4. The clinical radiologist is providing a medical report for the patient to assist in the management of the case and should interact closely with the primary care physician. It should be understood that the radiologist will recommend additional non requested examinations in a number of patients and the policy for progressing the diagnosis should be agreed with primary care. This may be affected by the financial system in place.

5. Delivery of imaging services within the primary care setting should be clinically and cost effective and delivered by properly trained personnel. It should be defined according to the organisation and goals of the service.

6. A financial system should be put in place to empower and encourage the provision of imaging services for primary care.

7. Teleradiology should be a vehicle to improve the direct support of the primary care physician - not an isolated reporting service. 
Acknowledgements The writing group was led by Prof Iain McCall from the ESR and Profs Manfred Maier, Christos Lionis and Eva Hummer-Pradier from WONCA Europe. The paper was approved by the ESR Executive Committee 2010.

\section{References}

1. World Organization of National Colleges, Academies and Academic Associations of General Practitioners/Family Physicians (World Organization of Family Doctors) WONCA Europe (2002) The European definition of General Practice/Family Medicine accessible at http://www.woncaeurope.org

2. Royal College of General Practitioners (2004) Radiology and the Patients of GPs: Joint statement of Royal College of General Practitioners and Royal College of Radiologists http://www.rcgp. org.uk/news_and_events/college_viewpoint/position_statements/ radiology_and the patients_of.aspx

3. Allen J, Gay B, Crebolder H, Heyrman J, Svab I, Ram P (2002) The European definitions of the key features of the discipline of general practice: the role of the GP and core competencies. Br J Gen Pract 52:526-527

4. Radiological Society of North America (2008) Multispecialty Collaboration Seen as Key to Radiology's Future. RSNA News http://www.rsna.org/Publications/rsnanews/June-2008/Multispe cialty_Collaboration_feature.cfm

5. Speets AM, van der Graaf Y, Hoes AW, Kalmijn S, Sachs AP, Rutten MJ, Gratama JW, Montauban van Swijndregt AD, Mali WP (2006) Chest radiography in general practice: indications, diagnostic yield and consequences for patient management. $\mathrm{Br} \mathrm{J}$ Gen Pract 56:574-578

6. Peat G, Thomas E, Duncan R, Wood L, Wilkie R, Hill J, Hay EM, Croft P (2007) Estimating the probability of radiographic osteoarthritis in the older patient with knee pain. Arthritis Rheum 57:794-802

7. Bedson J, Jordan KP, Croft PR (2007) A cross sectional study of requests for knee radiographs from primary care. BMC Musculoskelet Disord 8:77-85

8. White PM, Halliday-Pegg JC, Collie DA (2002) Open access neuroimaging for general practitioners-diagnostic yield and influence on patient management. Br J Gen Pract 52:33-35
9. Miller P, Kendrick D, Bentley E, Fielding K (2002) Costeffectiveness of lumbar spine radiography in primary care patients with low back pain. Spine 27:2291-2297

10. Jarvik JG, Hollingworth W, Martin B, Emerson SS, Gray DT, Overman S, Robinson D, Staiger T, Wessbecher F, Sullivan SD, Kreuter W, Deyo RA (2003) Rapid magnetic resonance imaging vs radiographs for patients with low back pain: a randomized controlled trial. JAMA 289:2810-2818

11. Andronis L, Atwell C, Brealey S, DAMASK (Direct Access to Magnetic Resonance Imaging: Assessment for Suspect Knees) Trial Team et al (2008) Cost-effectiveness of magnetic resonance imaging of the knee for patients presenting in primary care. Br J Gen Pract 58:e10-6

12. Royal College of Radiologists Working Party (1993) Influence of Royal College of Radiologists' guidelines on referral from general practice. BMJ 306:110-111

13. The Council of the European Union (2007) Council Directive 97/ 43/Euratom of 30 June 1997 on health protection of individuals against the dangers of ionizing radiation in relation to medical exposure http://eur-lex.europa.eu/LexUriServ/LexUriServ.do? uri=CELEX:31997L0043:EN:HTML

14. Berendsen AJ, Benneker WH, Schuling J, Rijkers-Koorn N, Slaets JP, Meyboom-de Jong B (2006) Collaboration with general practitioners: preferences of medical specialists-a qualitative study. BMC Health Serv Res 6:155

15. Glazebrook R, Chater B, Graham P, Kokar G, Robinson M, Escott R, Putman J, Crosbie D (2005) Evaluation of the ACRRM national radiology program for Australian rural and remote medical practitioners. Rural Remote Health 5:349

16. European Commission (2001) Referral guidelines for imaging. European Commission ISBN 92-828-9454-1 http://ec.europa.eu/ energy/nuclear/radioprotection/publication/doc/118 en.pdf

17. The Royal College of Radiologists (2007) Making the best use of Clinical Radiology Services: Referral Guidelines (sixth edition). The Royal College of Radiologists; London ISBN 978-1-90503424-6

18. Molinari G, Valbusa A, Terrizzano M, Bazzano M, Torelli L, Girardi N, Barsotti A (2004) Nine years' experience of telecardiology in primary care. J Telemed Telecare 10:249-253

19. Mavrogeni S, Sotiriou D, Thomakos D, Venieris N, Panagopoulos P (1996) Telecardiology services in the Aegean islands. J Telemed Telecare 2(Suppl 1):74-76 